\title{
Optical phonons of strained GaAs/GaP quantum wells studied by Raman spectroscopy
}

\author{
P. Castrillo \\ Departamento E. y Electrónica, Universidad de Valladolid, Doctor Mergelina s/n, 47011 Valladolid, Spain \\ and Department of Solid State Physics, Lund University, 22100 Lund, Sweden \\ G. Armelles, J. P. Silveira, and F. Briones \\ Instituto de Microelectrónica de Madrid, CSIC, Isaac Newton 8, 28760 Tres Cantos, Spain \\ J. Barbolla \\ Departamento E. y Electrónica, Universidad de Valladolid, Doctor Mergelina s/n, 47011 Valladolid, Spain
}

(Received 22 May 1997; accepted for publication 3 July 1997)

\begin{abstract}
We have studied the optical phonons of GaAs quantum wells strained to GaP. The phonon frequencies have been measured by Raman spectroscopy. The results have been compared with calculations based on the linear chain model and the random isoamplitude model. The comparison suggests a certain degree of atomic intermixing at the interfaces, mainly due to a limited but measurable arsenic carry-over during growth. (c) 1997 American Institute of Physics.

[S0003-6951(97)02036-6]
\end{abstract}

Although GaAs and GaP are two important materials in optoelectronics, very few investigations have been reported concerning pseudomorphic GaAs/GaP structures. In such a material combination there are growth limitations coming both from the lattice mismatch between $\mathrm{GaAs}$ and $\mathrm{GaP}$ $(3.6 \%)^{1}$ and from the competition between arsenic and phosphorus for the incorporation during the epitaxial growth. ${ }^{2}$ Only very recently strained GaAs quantum wells (QWs) in GaP with good optical properties have been achieved. ${ }^{3}$ These QWs are expected to also exhibit interesting phonon properties. The phonon dispersion curves for $\mathrm{GaP}$ are very similar to those for $\mathrm{AlAs}^{4}$ and, therefore, as far as the phonon properties are concerned, the main difference between $\mathrm{GaAs} / \mathrm{GaP}$ QWs and GaAs/AlAs QWs is the effect that the built-in strain has on the pseudomorphic GaAs layers. Thus, Raman spectroscopy in such $\mathrm{GaAs} / \mathrm{GaP} \mathrm{QWs}$ would provide a rare opportunity to study the properties of confined phonons in highly strained GaAs. In the present letter we report a Raman study concerning the combined effect of strain and confinement on the optical phonons of strained GaAs QWs in GaP. Additionally, the effect on these phonons of atomic intermixing at the interfaces is analyzed and the actual composition profiles are discussed.

The samples were grown by atomic layer molecular beam epitaxy $(\mathrm{ALMBE})^{5}$ on $\{001\}$-oriented GaP substrates at a substrate temperature of $400{ }^{\circ} \mathrm{C}$ using fast switching valved cracker cells both for arsenic and phosphorus. A previous $\mathrm{GaP}$ buffer was grown by molecular beam epitaxy (MBE). The structures consist of three GaAs QWs separated by 38nm-thick $\mathrm{GaP}$ spacing layers. The GaAs layer thickness, $d_{\mathrm{GaAs}}$, has been varied from sample to sample in steps of one monolayer (ML) covering the range from 1 to 6 MLs. The critical thickness for the growth of $\mathrm{GaP}$ on GaAs in our growth conditions is 6 MLs. ${ }^{6}$ The corresponding value for $\mathrm{GaAs}$ on $\mathrm{GaP}$ has not been measured, but it is expected to be similar. In fact, neither three-dimensional growth nor appreciable roughness could be inferred from the reflection highenergy electron diffraction (RHEED) patterns during the growth of the samples. Additionally, no degradation of the optical properties and no indication of the existence of is- lands were obtained in the ex situ optical characterization. ${ }^{3}$ Therefore, the GaAs QWs are expected to be free of threedimensional islands and dislocations. Hence, they can be considered to be biaxially strained with an in-plane lattice parameter equal to that of the GaP substrate.

Raman spectra were obtained at $80 \mathrm{~K}$ using a triple spectrometer incorporating a charge-coupled device (CCD) camera system. The strained GaAs QWs studied here show absorption-related electronic transitions in the energy range between 2.1 and $2.7 \mathrm{eV}$, depending on the quantum well thickness. ${ }^{3}$ Therefore we were able to excite either in or outside resonance conditions by using the different lines of an $\mathrm{Ar}^{+}$laser. The spectra were recorded in two different backscattering geometries, $z(x x) \bar{z}$ and $x(x y) \bar{z}$, being $\{x, y, z\}$ $=\{[100],[010],[001]\}$. The contribution of the GaAs QWs to the Raman spectra outside resonance conditions mainly appears in the $z(x y) \bar{z}$ configuration and is negligible in the $z(x x) \bar{z}$ configuration. Under near resonance conditions the contribution of the GaAs QWs to the $z(x x) \bar{z}$ spectra increases and the $z(x x) \bar{z}$ and $z(x y) \bar{z}$ spectra become very similar in the range of the GaAs phonons.

Figures 1 show the Raman spectra taken in the $z(x y) \bar{z}$ and $z(x x) \bar{z}$ in the frequency range of the GaAs-related optical phonons. The excitation wavelengths for the spectra of Fig. 1(a) were chosen to be outside resonance conditions, whereas in the case of Fig. 1(b) they were chosen to be near or in resonance with an electronic transition of the GaAs QWs. In Fig. 1(a) $[z(x y) \bar{z}$ geometry] only one Raman peak is observed, which corresponds to the first confined longitudinal optical phonon $\left(\mathrm{LO}_{1}\right)$ of the GaAs layers. In Fig. 1(b) $[z(x x) \bar{z}$ geometry] we observe two Raman peaks. The peak located at higher frequency corresponds to the second confined longitudinal optical phonon $\left(\mathrm{LO}_{2}\right)$ of the GaAs layers. The peak located at lower frequency is attributed to an interface mode (IF) of the type described in Ref. 7. For the sample with $d_{\mathrm{GaAs}}=6 \mathrm{MLs}$ such interface mode is immersed in a band of the GaP substrate. For the sample with $d_{\mathrm{GaAs}}=1 \mathrm{ML}$ we were not able to obtain any signal related to the GaAs layers in the $z(x x) z$ spectrum. The experimental frequencies of the $\mathrm{LO}_{1}$ and $\mathrm{LO}_{2}$ peaks are displayed in Fig. 2 


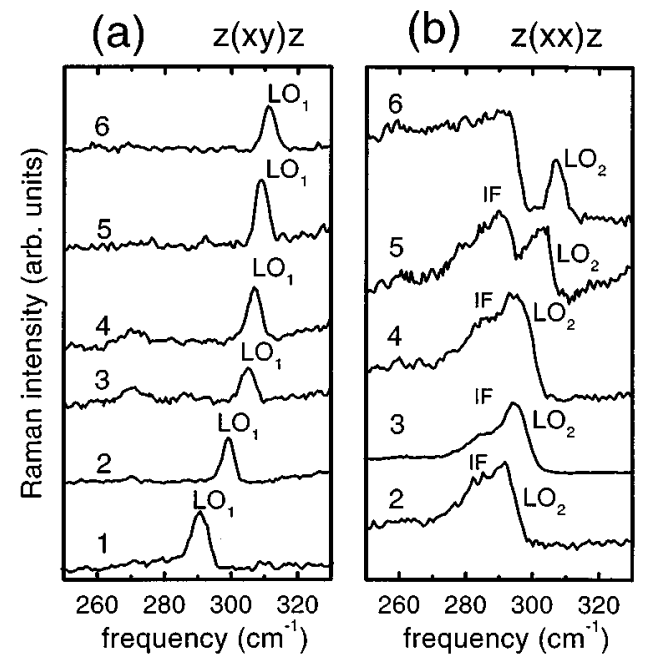

FIG. 1. Raman spectra of strained GaAs/GaP QWs (a) in the $z(x y) \bar{z}$ geometry outside resonance conditions and (b) in the $z(x x) \bar{z}$ geometry under resonance conditions. The label of each spectrum refers to the QW thickness (given in monolayers).

(symbols) as a function of the GaAs layer thickness. The effect of confinement is clearly seen in the figure: when the layer thickness decreases the frequency of the phonons $\left(\mathrm{LO}_{1}\right.$ and $\mathrm{LO}_{2}$ ) also decreases. The effect of the in-plane strain is also observed: the $\mathrm{LO}_{1}$ peak of the $6 \mathrm{MLs}$ sample is located at $311 \mathrm{~cm}^{-1}$, about $16 \mathrm{~cm}^{-1}$ higher than the LO frequency of unstrained GaAs.

In order to quantitatively analyze the role of the strain and the confinement we have calculated the phonon frequencies of these QWs. For unstrained III-V binary compounds a simple linear chain model with interaction up to second neighbors gives a good description of the longitudinal modes propagating along the [001] direction. The three force constant parameters of the model can be determined from the experimental frequencies of the longitudinal modes of bulk material at the points $\Gamma$ and $X$ of the Brillouin zone $\left[\omega_{\mathrm{LO}}(\Gamma)\right.$,

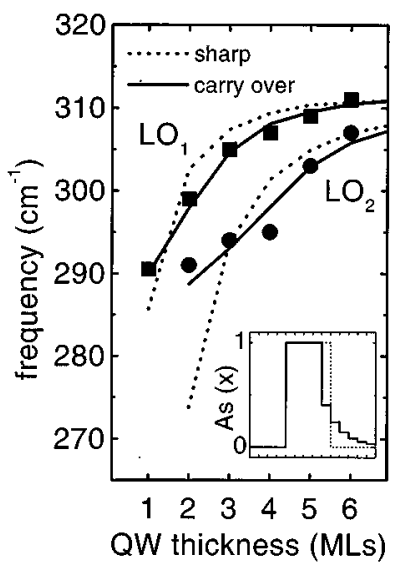

FIG. 2. Experimental and calculated frequencies of confined phonons in strained GaAs QWs as a function of the QW thickness (in monolayers, MLs). The experimental frequencies of $\mathrm{LO}_{1}$ and $\mathrm{LO}_{2}$ phonons are represented by symbols (squares and dots, respectively). Dashed lines correspond to the calculated frequencies assuming sharp interfaces while solid lines correspond to a carry-over of As with a segregation length of 2 MLs. The profiles of As concentration assumed in both cases are displayed in the inset for a 5-ML-thick QW. (Each tick in the abscises of the inset corresponds to $1 \mathrm{ML}$.
$\omega_{\mathrm{LO}}(X)$ and $\left.\omega_{\mathrm{LA}}(X)\right]^{4,8}$ The strain can be taken into account by a modification of the force constants in terms of the phonon deformation potential, the elastic constants, and the built-in strain. ${ }^{9}$ The strain-induced modification of the firstneighbor force constants can be given by

$$
K_{\mathrm{A}-\mathrm{B}}(\epsilon)=K_{\mathrm{A}-\mathrm{B}}(0) \cdot(1+\sigma \cdot \epsilon),
$$

where $K_{\mathrm{A}-\mathrm{B}}(0)$ denotes the force constant the $\mathrm{A}$ and $\mathrm{B}$ planes for the unstrained material, $\epsilon$ the in-plane strain $[\epsilon$ $\left.=\left(a_{\mathrm{GaP}}-a_{\mathrm{GaAs}}\right) / a_{\mathrm{GaAs}}\right]$ and $\sigma=-2\left(k_{12}-k_{11} \cdot C_{12} / C_{11}\right)$. The coefficients $k_{i j}$ are the phonon deformation potentials as defined in Ref. 10, and $C_{i j}$ denote the elastic moduli. We assume that the other two force constants $\left(K_{\mathrm{A}-\mathrm{A}}\right.$ and $\left.K_{\mathrm{B}-\mathrm{B}}\right)$ vary in the same proportion as $K_{\mathrm{A}-\mathrm{B}}$. Within this assumption the strain produces a nearly rigid shift of the phonon dispersion curve, a result similar to the one obtained using more elaborated models. ${ }^{11}$ For the calculations of the LO phonon frequencies of strained GaAs QWs we built a supercell using the force constants of strained GaAs and unstrained $\mathrm{GaP}^{12}$ The frequencies of the IF phonons cannot be calculated within this model and they will not be analyzed here.

Figure 2 displays in dashed lines the calculated frequencies of the $\mathrm{LO}_{1}$ and $\mathrm{LO}_{2}$ phonons confined in the GaAs QWs as a function of the thickness. The calculated frequencies are resulting from both the effect of the strain, which induces a shift of $17 \mathrm{~cm}^{-1}$ to higher frequencies, and the effect of the confinement, which induces a shift to lower frequencies that depends on $d_{\mathrm{GaAs}}$. As it can be seen in Fig. 2, the calculated and the experimental values are in good agreement for the sample of thickest QWs ( $\left.d_{\mathrm{GaAs}}=6 \mathrm{MLs}\right)$, in which the effect of confinement is weak. Therefore, such an agreement suggests that the strain contribution has been properly assessed and, in turn, corroborates that GaAs layers are pseudomorphically strained. In contrast the discrepancies are noticeable in the case of thinner QWs. In fact, for decreasing QW thickness, the experimental frequencies of $\mathrm{LO}_{1}$ and $\mathrm{LO}_{2}$ phonons are initially lower than the calculated values, this situation being inverted for even thinner QWs. A similar behavior has been reported for the phonon frequencies of GaAs/AlAs QWs in Ref. 13. Therein, such a down-shift of highfrequency phonons and up-shift of low-frequency phonons was shown to be induced by the existence of atomic intermixing at the interfaces. In order to verify if the As-P intermixing can account for our experimental results we have included this effect in the model and we have performed calculations with different concentration profiles for the alloy at the interfaces.

$\mathrm{GaAs}_{x} \mathrm{P}_{1-x}$ alloys have a two mode behavior ${ }^{14}$ and they can be described using the random isoamplitude model. ${ }^{15}$ As a generalization of the model for binary compounds we have included force constants up to second neighboring planes. Therefore, we have now two first-neighbor force constants $\left(K_{\mathrm{Ga}-\mathrm{As}}, K_{\mathrm{Ga}-\mathrm{P}}\right)$ and four second-neighbor force constants $\left(K_{\mathrm{Ga}-\mathrm{Ga}}, K_{\mathrm{As}-\mathrm{As}}, K_{\mathrm{P}-\mathrm{P}}\right.$, and $\left.K_{\mathrm{As}-\mathrm{P}}\right)$. As in Ref. 15 , the force constants vary linearly with the composition $x$. The linear dependence of $K_{\mathrm{Ga}-\mathrm{As}}(x), K_{\mathrm{Ga}-\mathrm{P}}(x)$, and $K_{\mathrm{As}-\mathrm{P}}(x)$ are determined from the frequencies of the local modes of As in $\mathrm{GaP}$ and $\mathrm{P}$ in $\mathrm{GaAs}^{16,17}$ and from the frequencies of GaAslike and GaP-like LO modes in unstrained $\mathrm{GaAs}_{x} \mathrm{P}_{1-x} \cdot{ }^{17,18}$ $K_{\mathrm{Ga}-\mathrm{Ga}}(x)$ varies between the values of the two binary com- 
pounds. $K_{\mathrm{As}-\mathrm{As}}(x)$ and $K_{\mathrm{P}-\mathrm{P}}(x)$ are assumed to change in the same proportion than $K_{\mathrm{Ga}-\mathrm{As}}(x)$ and $K_{\mathrm{Ga}-\mathrm{P}}(x)$, respectively. The strain modifies the force constants as in the binary compounds [Eq. (1)] with the only difference that the strain coefficients $\sigma_{\mathrm{GaAs}}(x)$ and $\sigma_{\mathrm{GaP}}(x)$ depend on the composition. ${ }^{19,20}$ The model described for alloys can be extended to QWs with intermixing. However in this case both the composition and strain, and thus the force constants, vary locally in the supercell.

Using this model, we have performed simulations for different concentration profiles corresponding to three major mechanisms occurring during the growth that can be responsible for the As-P intermixing in the QW structures: ${ }^{21-24}$

(i) short-range interdiffusion,

(ii) phosphorus segregation, and

(iii) arsenic segregation ("carry-over").

In the first two extreme cases (i) and (ii) it was not possible to achieve a good fitting for the frequencies of both $\mathrm{LO}_{1}$ and $\mathrm{LO}_{2}$ phonons. In contrast, a noteworthy improvement of the fitting could be obtained in case (iii), in which the concentration profile results from a carry-over of arsenic from the topmost GaAs monolayer assuming the lowest interface to be sharp. The best agreement with the experiment was achieved assuming a segregation length of 2 MLs (i.e., a segregation coefficient ${ }^{22}$ of about 0.6 ). The results of this calculation are included in Fig. 2 (solid lines). The corresponding As concentration profile is displayed in the inset. Besides the simplicity of the model, the general trend of the experimental frequencies is very well reproduced. Moreover, within this picture, the small features observed in Fig. 1(a) around $272 \mathrm{~cm}^{-1}$ are attributed to local modes of As in GaP and they would be related to a small amount of As atoms segregated a few monolayers away from the QWs.

According to the previous discussion, the experimental results can be explained assuming an arsenic segregation, that would be produced by an As-P exchange at the surface of the growing sample. Short range interdiffusion and phosphorus segregation could play a minor role. This is consistent with the expected behavior for the growth conditions. On one hand the interdiffusion mechanism is too weak at low growth temperatures to be significant. ${ }^{21}$ On the other hand the segregation coefficient increases with the atomic number $^{22}$ and, hence, As segregation is expected to be stronger than P segregation. Moreover, in our case the As carryover might be enhanced due to the high compressive strain related to GaAs inside the GaP lattice. The fitting in Fig. 2 is slightly improved when a weak broadening of the lowest interface is included but this effect seems to be weak and no conclusive information can be drawn. Finally, compared to Raman studies in other material systems, the degree of intermixing in our samples is of the same order as in high temperature-grown AlAs/GaAs structures but higher than in AlAs/GaAs structures grown at low temperature. ${ }^{13,21}$ However, the segregation length that we found is smaller than those reported in the literature for the highly strained InAs/ GaAs system. ${ }^{22}$

In summary, a set of GaAs QWs strained to GaP with thicknesses ranging between 1 and 6 MLs has been studied by Raman spectroscopy. The phonon frequencies have been calculated within a phenomenological scheme including the effects of strain and of a possible intermixing at the interfaces. The comparison between theory and experiments suggests that the strain has been properly assessed but that there is a certain degree of As-P intermixing. Remarkable agreement between calculated and experimental frequencies is obtained when arsenic segregation (carry-over) at the upper interface is assumed. The results are consistent with a segregation length of about 2 MLs.

The authors would like to thank M. S. Miller, M.-E. Pistol, A. Postigo, and Professor L. Samuelson for helpful discussions. This work has been partially supported by the Spanish CICYT under project No. MAT95-0966-c02-01 and by the Human Capital and Mobility project HYPOCRATES.

${ }^{1}$ T. P. Pearsall, ' Strained-Layer Superlattices: Physics,', in Semiconductor and Semimetals, edited by R. K. Willardson and A. C. Beer (Academic, New York, 1990), Vol. 32.

${ }^{2}$ C. T. Foxon, B. A. Joyce, and M. T. Norris, J. Cryst. Growth 49, 774 (1980)

${ }^{3}$ J. A. Prieto, G. Armelles, M.-E. Pistol, P. Castrillo, J. P. Silveira, and F. Briones, Appl. Phys. Lett. 70, 3449 (1997).

${ }^{4}$ Numerical Data and Functional Relationships in Science and Technology, Landolt-Börnstein New Series, Group III, edited by O. Madelung (Springer, Berlin, 1982), Vol. 17a.

${ }^{5}$ F. Briones, L. González, and A. Ruiz, Appl. Phys. A 49, 729 (1989).

${ }^{6}$ A. Mazuelas, L. González, F. A. Ponce, L. Tapfer, and F. Briones, J. Cryst. Growth 131, 465 (1993).

${ }^{7}$ A. K. Sood, J. Menéndez, M. Cardona, and K. Ploog, Phys. Rev. Lett. 54, 2115 (1985).

${ }^{8}$ D. Strauch and B. Dorner, J. Phys.: Condens. Matter 2, 1457 (1990).

${ }^{9}$ M. I. Alonso, P. Castrillo, G. Armelles, A. Ruiz, M. Recio, and F. Briones, Phys. Rev. B 45, 9054 (1992).

${ }^{10}$ P. Wickbolt, E. Anastassakis, R. Sauer, and M. Cardona, Phys. Rev. B 35, 1362 (1987)

${ }^{11}$ A. Qteish and E. Molinari, Phys. Rev. B 42, 7090 (1990).

${ }^{12}$ The force constants that we have used are: $K_{\mathrm{Ga}-\mathrm{P}}=103.61, K_{\mathrm{Ga}-\mathrm{Ga}}$ $=9.83$, and $K_{\mathrm{P}-\mathrm{P}}=10.64 \mathrm{~N} / \mathrm{m}$ for unstrained $\mathrm{GaP}$; and: $K_{\mathrm{Ga}-\mathrm{As}}=92.57$, $K_{\mathrm{Ga}-\mathrm{Ga}}=12.86$, and $K_{\mathrm{As}-\mathrm{As}}=9.57 \mathrm{~N} / \mathrm{m}$, for unstrained GaAs. The value of the strain coefficient of GaAs, taken from Ref. 10, is $\sigma_{\mathrm{GaAs}}=-3.3$.

${ }^{13}$ D. Gammon, B. V. Shanabrook, and D. S. Katzer, Phys. Rev. Lett. 67, 1547 (1991).

${ }^{14}$ Y. S. Chen, W. Shockley, and G. L. Pearson, Phys. Rev. 151, 648 (1955).

${ }^{15}$ J. Leng, Y. Quian, P. Chen, and A. Madhukar, Solid State Commun. 69, 311 (1989).

${ }^{16}$ A. S. Barker, Jr., Phys. Rev. 165, 917 (1968); W. G. Spitzer, J. Phys. Chem. Solids 28, 33 (1967)

${ }^{17}$ M.-E. Pistol and X. Liu, Phys. Rev. B 45, 4312 (1992).

${ }^{18}$ The fitting force constants that we have used are: $K_{\mathrm{Ga}-\mathrm{As}}(x=0)$ $=152.74, K_{\mathrm{Ga}-\mathrm{P}}(x=1)=97.39, K_{\mathrm{As}-\mathrm{P}}(x=0)=10.61$, and $K_{\mathrm{As}-\mathrm{P}}(x=1)$ $=16.16 \mathrm{~N} / \mathrm{m}$. The other force constants are obtained from the binary compounds. With this set of parameters a good description of the alloy frequencies is obtained for the full range of compositions.

${ }^{19}$ G. Armelles, M. L. Sanjuán, L. González, and Y. González, Appl. Phys. Lett. 68, 1806 (1996).

${ }^{20}$ In order to fit the experimental data for $\mathrm{GaAs}_{x} \mathrm{P}_{1-x}$ alloys reported in Ref. 19 we have taken $\sigma_{\mathrm{GaAs}}(x)$ and $\sigma_{\mathrm{GaP}}(x)$ to be linear, being $d \sigma_{\mathrm{GaAs}} / d x$ $=8.5$ and $d \sigma_{\mathrm{GaP}} / d x=2.4$, and being $\sigma_{\mathrm{GaAs}}(x=1)=-3.3, \sigma_{\mathrm{GaP}}(x=0)$ $=-3.7$.

${ }^{21}$ B. Jusserand, F. Alexandre, D. Paquet, and G. Le Roux, Appl. Phys. Lett. 47, 301 (1985).

${ }^{22}$ J. M. Moison, C. Guille, F. Houzay, F. Barthe, and M. Van Rompay, Phys. Rev. B 40, 6149 (1989).

${ }^{23}$ L. Samuelson and W. Seifert, in Handbook of Crystal Growth, edited by D. T. J. Hurle (Elsevier Science B.V., Amsterdam, 1994), Vol. 3, p. 747.

${ }^{24}$ B. Jusserand, F. Mollot, J. M. Moison, and G. Le Roux, Appl. Phys. Lett. 57, 560 (1990); 61, 423 (1992). 